\title{
K-RAS4A: Lead or Supporting Role in Cancer Biology?
}

\section{Veronica Aran* \\ Laboratorio de Biomedicina Do Cérebro, Instituto Estadual Do Cérebro Paulo Niemeyer, Rio de Janeiro, Brazil}

The RAS oncogene is one of the most frequently mutated genes in human cancer, with K-RAS having a leading role in tumorigenesis. K-RAS undergoes alternative splicing, and as a result its transcript generates two gene products K-RAS4A and K-RAS4B, which are affected by the same oncogenic mutations, are highly homologous, and are expressed in a variety of human tissues at different levels. In addition, both isoforms localise to the plasma membrane by distinct targeting motifs. While some evidence suggests nonredundant functions for both splice variants, most work to date has focused on K-RAS4B, or even just K-RAS (i.e., without differentiating between the splice variants). This review aims to address the most relevant evidence published regarding K-RAS4A and to discuss if this "minor" isoform could also play a leading role in cancer, concluding that a significant body of evidence supports a leading role rather than a supporting (or secondary) role for K-RAS4A in cancer biology.

\section{OPEN ACCESS}

Edited by:

Mahendra Pratap Kashyap, University of Alabama at Birmingham, United States

Reviewed by: Sangwoo Kim,

Yonsei University, South Korea Avaniyapuram Kannan Murugan, King Faisal Specialist Hospital and Research Centre, Saudi Arabia

${ }^{*}$ Correspondence: Veronica Aran varanponte@gmail.com

Specialty section: This article was submitted to Molecular Diagnostics and Therapeutics,

a section of the journal Frontiers in Molecular Biosciences

Received: 23 June 2021 Accepted: 01 September 2021 Published: 15 September 2021

Citation:

Aran V (2021) K-RAS4A: Lead or Supporting Role in Cancer Biology?

Front. Mol. Biosci. 8:729830. doi: 10.3389/fmolb.2021.729830
Keywords: K-ras, K-Ras4A, K-Ras4B, alternative splicing, cancer

\section{INTRODUCTION}

The importance of gene alternative splicing has been well documented. This conserved biological process occurs when a single gene produces different mRNA transcripts, thus helping to contribute to the formation of a vast transcriptome and proteome (Kelemen et al., 2013). This process generates protein diversity, as a single gene can result in the production of different variants of a protein, which may exhibit differential tissue expression (Sorek and Amitai 2001). In summary, alternative splicing results in different: 1) protein function; 2) tissue expression; 3) localisation; enzymatic activities; and 4) protein-protein interactions (Kelemen et al., 2013). The differences between splice variants are of pharmaceutical importance since they may contribute to variable treatment responses.

There are three RAS genes encoding four isoforms, which are ubiquitously expressed in human cells and share $82-90 \%$ sequence homology. These four isoforms are H-RAS, N-RAS, K-RAS4A and K-RAS4B (Cox and Der 2010). RAS mutations are frequently found in cancer ( $24 \%$ of all cancers) (Stalnecker and Der 2020), where the K-RAS gene is mutated in approximately $17 \%$ of all cancer types (46,213 mutant samples/272047 samples tested), N-RAS gene is mutated in $\sim 5.1 \%(7,926$ mutant samples/154172 samples tested), and H-RAS in 2.3\% (2,404 mutant samples/106318 samples tested) (as reported in the Catalog of Somatic Mutated in Cancer, COSMIC database, v94, in August 2021). RAS mutations are crucial for personalised medicine since they can direct targeted therapies and serve as diagnostic and prognostic markers for different cancers (Murugan et al., 2019). In fact, K-RAS mutations were considered adverse prognostic factors and indicators of EGFRtargeted therapy resistance in certain cancer types such as lung and colorectal (Pao et al., 2005; Marks et al., 2008; Normanno et al., 2009). Figure 1 summarizes some of the most frequently K-RAS mutated tissues based on the COSMIC database (searched in COSMIC database, v94, in May 2021).

The discovery over 35 years ago (McGrath et al., 1983; Shimizu et al., 1983) of the fourth exons 4A and $4 \mathrm{~B}$ resulted in the identification of the existence of two protein isoforms, K-RAS4A and K-RAS4B [189 and 


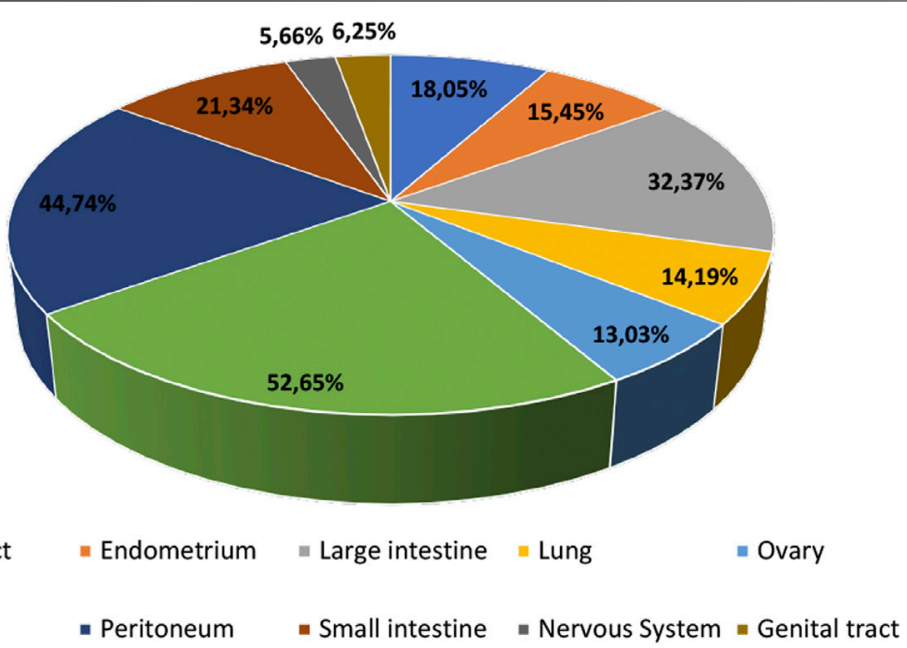

FIGURE 1 | Most frequent human tissues affected by K-RAS mutations based on the COSMIC database, v94 (data obtained in May 2021).

188 amino acids (aa), respectively]. The 21-kDa RAS gene products shares $100 \%$ sequence homology in the first 86 aa residues among different RAS isoforms (K-, N- and H-RAS) (Messina et al., 2019). The RAS G domain comprises the first 165 aa, representing the catalytic and switching region where the exchange between GDP/ GTP occurs. It is also the domain to which different effectors, exchange factors, and GTPase-activating proteins (GAPs) bind. K-RAS plays an essential role in mouse embryonic development (Koera et al., 1997; Hobbs, Der, and Rossman 2016), whereas K-RAS4A, H-RAS and N-RAS expression is dispensable for mouse development (Esteban et al., 2001; Plowman et al., 2003). Unlike K-RAS4A, K-RAS4B has been heavily researched.

Mutations that activate K-RAS usually affect codons 12, 13, and 61 (the majority being missense substitutions), which are common to both genes, thus rendering both oncogenic (Capon et al., 1983). The biological relevance of the alternative splicing of K-RAS has never been fully elucidated. Most studies have concentrated their attention on K-RAS4B rather than K-RAS4A. For example, while a Pubmed search on "K-RAS" or "KRAS" yields 21,408 results, a search on "KRAS4A" or "KRAS4A" or "K-Ras4A" or "KRas4A" or "K-Ras4a" or "KRas4a" yields 54 results and a search on "K-RAS4B" or "KRAS4B" or "K-Ras4B" or "KRas4B" or "K-Ras4B" or "K-Ras4b" or "KRas4b" yields 213 results (all searched on May 20, 2021). This finding suggests that most studies have not discriminated by K-RAS isoform. Nevertheless, the two splice variants exhibit differential tissue expression (Newlaczyl et al., 2017). Therefore, the present review aims to improve the general understanding of each isoform by describing previous work an discussing potential roles of K-RAS4A in cancer.

\section{K-RAS4A VERSUS K-RAS4B: STRUCTURE AND SIGNALLING}

It is well stablished that RAS isoforms exhibit distinct biological activities and subcellular localisations that depend mainly on the interaction between the C-terminal hypervariable region (HVR) and host membranes (Hancock 2003; Laude and Prior 2008). The
HVR region is composed of a linker domain comprising aa 166-178/179, and a targeting domain comprising aa 179/ 180-189/188, which undergoes posttranslational modifications that mediate membrane binding. The HVR contains a C-terminal CAAX (CAAX motif) sequence, which is modified posttranslationally (Wright and Philips 2006). The C-terminal cysteine is farnesylated for weak membrane interaction; further membrane binding stabilisation requires a second signal within the HVR region (Hancock et al., 1991). For K-RAS4B, this signal is electrostatic (i.e., six contiguous lysines), whereas for the other RAS isoforms (K-RAS4A, H-RAS and N-RAS), this stabilisation is mediated by palmitoylation (Hancock et al., 1990). The isoform H-RAS contains two palmitoylation sites within the HVR region, whereas N-RAS and K-RAS4A are monopalmitoylated (Zhou et al., 2018). Additionally, K-RAS4B displays a unique feature, a phosphorylation site (aa S181) that behaves as an electrostatic farnesyl switch, inducing K-RAS4B translocation from the plasma membrane to other endomembrane compartments (Barcelo et al., 2014). The different posttranslational modifications that occur in the RAS C-terminal region were, and still are, considered potential targets for anti-cancer therapies despite the failure of farnesyltransferase inhibitors in the past (James, Goldstein, and Brown 1996; Konstantinopoulos et al., 2007; Ahearn et al., 2018).

RAS interaction with the plasma membrane is required for its function. K-RAS4A and K-RAS4B differ mainly in their C-terminal regions (Laude and Prior 2008; Tsai et al., 2015), which in the case of K-RAS4A, contains a site of palmitoylation and a bipartite polybasic region able to independently deliver K-RAS4A to the plasma membrane (Laude and Prior 2008; Tsai et al., 2015; Zhao et al., 2015). This indicates that, compared to other RAS proteins, K-RAS4A is the only one harbouring a dual membrane-targeting motif and that K-RAS4B is more positively charged and less hydrophobic than K-RAS4A. It has been proposed that the bipartite polybasic region alongside the monopalmitoylation and farnesylation of K-RAS4A may affect its function and expression, in addition to place this variant 
between K-RAS4B and N-RAS in terms of protein similarities (Laude and Prior 2008; Nussinov et al., 2016).

Structural analysis using atom molecular dynamics simulations investigated K-RAS4A placement at membranes that contain anionic lipids (POPS or PIP2) (Li and Buck 2017). This study demonstrated that K-RAS4A prefers different orientations at the membrane, where both its topology and the electrostatic interaction between its charged residues and the anionic lipids influence its orientation (Li and Buck 2017). Hancock and others reported that inhibition of acid sphingomyelinase mislocalises K-RAS4A and K-RAS4B from the plasma membrane to the endomembrane and blocks their nanoclustering, thus suggesting that an indirect inhibitor of sphingomyelinase could serve as a potential anti-K-RAS agent (Cho et al., 2016).

The protein conformations of K-RAS4A and K-RAS4B have also been compared by all-atom molecular dynamics simulations to identify isoform-specific differences. The results suggested that the catalytic domain of GDP-bound K-RAS4A differs from that of K-RAS4B by presenting a more exposed nucleotide binding pocket, also showing distinct dynamic fluctuations in switch I and II regions, which could affect the interaction between the catalytic domain and downstream effectors (Chakrabarti et al., 2016).

All four RAS isoforms have been shown to possess different biological activities and effector signalling. At least 11 different RAS effector families have been described, which drive distinct signalling cascades (Hobbs et al., 2016). Although all RAS proteins can differentially activate the Raf-MEK-ERK signalling pathway and affect cell phenotype in vitro, K-RAS4A and K-RAS4B have been shown to differentially affect Raf-1 (Voice et al., 1999). Furthermore, application of stable isotope labelling with amino acids in cell culture (SILAC) and affinity-purification mass spectrometry (AP-MS) to characterize the nucleotide-dependent protein interactomes of K-RAS4A and K-RAS4B revealed novel interactomes for each variant, with comparable numbers of interacting proteins for both wildtype and mutant versions of each splice variant (Zhang et al., 2018). Zhang and others described that K-RAS4A interacts with Raf-1 with higher affinity than K-RAS4B, leading to increased RAF1-1MEK-ERK signalling cascade, and that K-RAS4A showed increased anchorage-independent growth in assays that compared K-RAS4A- and K-RAS4B-transformed NIH 3T3 cells (Zhang et al., 2018). Interestingly, Bigenzahn and others performed proteomic analysis using K-562 chronic myeloid leukaemia cell lines. They reported that, while the two RAS isoforms share 28 interactors, they also each have distinct interactomes, with K-RAS4A specifically binding to fewer proteins than K-RAS4B (15 proteins versus 29, respectively) (Bigenzahn et al., 2018). Cumulatively, these findings suggest a certain degree of functional overlap and also raise the possibility that the splice variants cooperate with each another or compensate for each other's function, depending on the cell type and intracellular pathway involved.

K-RAS4A protein was identified as a defattyacylation substrate of SIRT2, a member of the sirtuin family of protein lysine deacylases (Jing et al., 2017). Through biochemical and cell biology approaches, Jing and others found that K-RAS4A is fatty acylated on lysine residues at its C-terminal HVR, and that SIRT2 removes lysine fatty acylation from K-RAS4A, resulting in increased endomembrane localisation, interaction with A-Raf, and in turn enhanced K-RAS4A transforming activity (Jing et al., 2017). Thus, the study of small molecules that could inhibit the defatty-acylation activity of sirtuins may have therapeutic potential. Spiegelman and others developed a SIRT2 inhibitor, named JH-T4, which was the first such inhibitor to enhance K-RAS4A lysine fatty acylation in vitro (Spiegelman et al., 2019). Although JH-T4 showed anti-cancer effects in cancer cells, it was also toxic to normal cells, suggesting a lack of cancer cell selectivity (Spiegelman et al., 2019). Thus, JH-T4, while potentially promising, awaits further improvements that may enhance its cancer cell selectivity.

Collectively, the studies suggest that RAS effector pathways may be differentially impacted by RAS structural conformation, localisation to membranes, and isoform-specific binding affinities, which may lead to variable signalling outputs. Figure 2 compares the K-RAS4A and K-RAS4B protein sequences, highlighting the important residues for membrane binding, and also the simplified schematic representation of the RAS pathway indicates that each isoform has its own binding affinities for different effectors, which may result in a variety of cell responses.

\section{COMPARISON OF K-RAS4A AND K-RAS4B TISSUE EXPRESSION PROFILES}

Profiling of K-RAS splice variant expression have shown that K-RAS4A and K-RAS4B expression levels differ across tissues. The K-RAS4A/4B expression ratio varies according to normal versus tumour tissues, as well as by tumour type analysed (e.g. lung, pancreas and colorectal cancer) (Plowman et al., 2003; Abubaker et al., 2009; Aran et al., 2018). For example, in patients with non-small cell lung cancer (NSCLC), K-RAS4B mRNA showed higher expression than K-RAS4A (Aran et al., 2018). In contrast, similar splice variant levels were reported in the colon (Pells et al., 1997). When the gene expression profiles of each RAS isoform were characterized in a full developmental time course mouse tissue panel, K-RAS4B expression was frequently higher than K-RAS4A (Newlaczyl et al., 2017). The findings suggested that K-RAS4A is the most dynamically regulated RAS isoform (upregulated in pre-term in stomach, intestine, kidney and heart) (Newlaczyl et al., 2017).

A quantitative RT-PCR assay has been developed to detect the splice junction region and thus measure variant expression in human cancer cell lines (Tsai et al., 2015). Of the 30 cell lines tested, the isoform K-RAS4A was expressed in all of them; with similar levels to that of K-RAS4B detected in 17 human colorectal tumours. Analysis with splice variant-specific antibodies supported this finding (Tsai et al., 2015). K-RAS4A showed higher expression in colon cancer and melanoma cell lines than in other cell lines tested (Tsai et al., 2015). Furthermore, there were no significant differences in the relative abundance of the two K-RAS mRNAs among cells that harboured wildtype versus mutant K-RAS. Another study showed that K-RAS4A was found to be expressed in both human renal cell carcinomas and human renal cell carcinoma cells lines, with its upregulation sensitive to aldosterone (King et al., 2014).

As previously mentioned, the K-RAS4A HVR sequence shares similarities with those of K-RAS4B and N-RAS. Nussinov and 


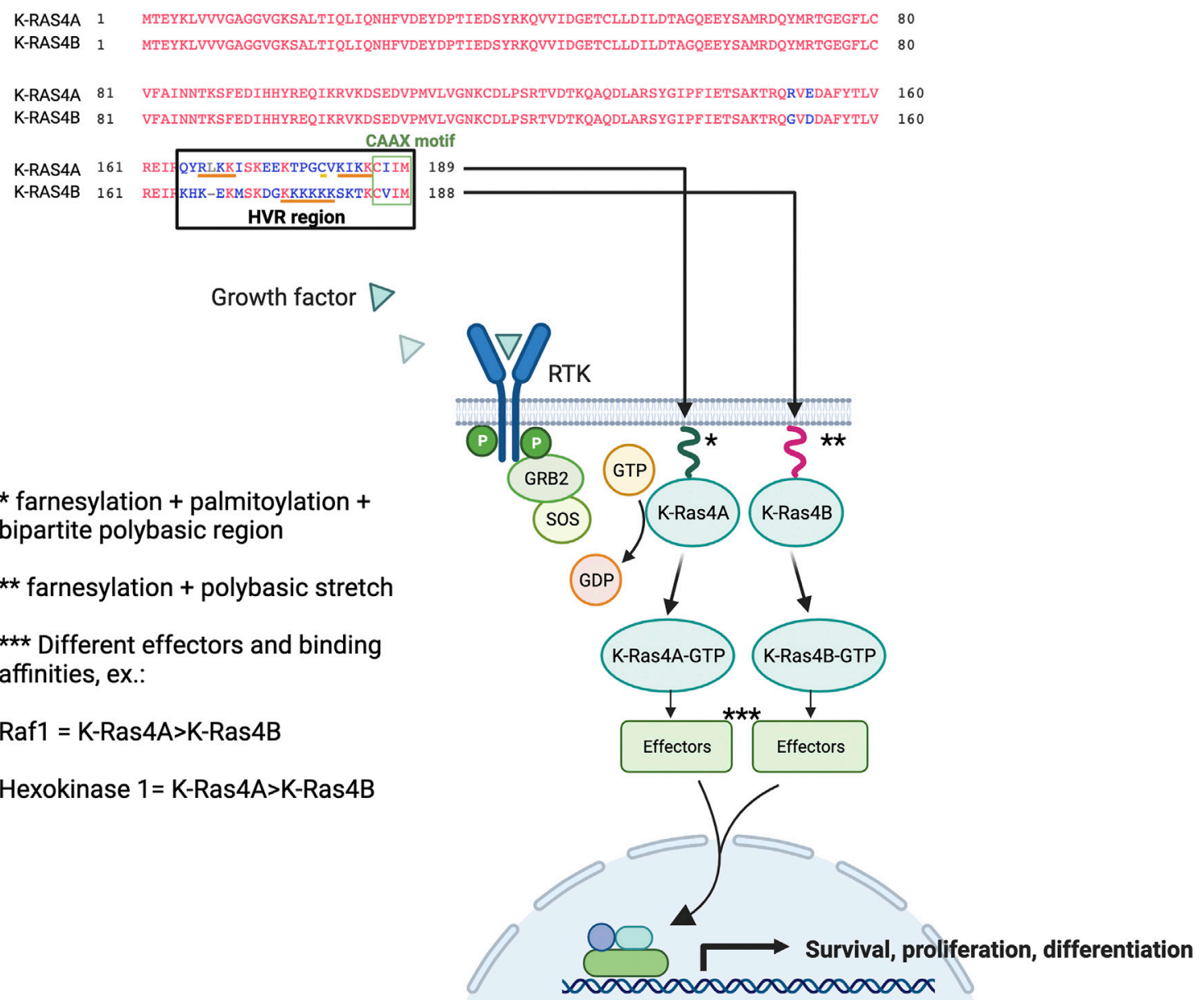

FIGURE 2 | K-RAS4A versus K-RAS4B protein sequence and signalling. RAS proteins contain a G domain (residues 1-165) responsible for the catalytic and switching portion of the protein that interacts with GDP/GTP, exchange factors, and GTPase-activating proteins (GAPs). The less homologous part corresponds to the hypervariable region (HVR) domains (final 23/24 residues). RAS are farnesylated on their C-terminal cysteine residue (CAAX motif), undergo AAX proteolysis and receive carboxyl methylation at the C-terminal prenylcysteine to allow the first step in membrane binding. A second motif improves this weak binding, which is a hexa-lysine polybasic stretch (residues 175-180) that interacts electrostatically with membranes in the case of K-RAS4B. Whereas, K-RAS4A membrane binding is stabilised by a monopalmitoylation site (residue 180), whereas this site is absent in the isoform K-RAS4B (Hancock et al., 1991). The KIKK motif (residues 182-185) was shown to be an additional membrane-targeting motif for K-RAS4A (Zhao et al., 2015), in addition to other basic motif corresponding to RLKK (residues 167-170) (Tsai et al., 2015). The binding of growth factors to the extracellular regions of receptor tyrosine kinases (RTKs) initiates the signal that will lead to the activation of RAS proteins downstream of the receptor. The isoforms can bind to a variety of effectors with variable affinities where, for example, hexokinase 1 was shown to bind to K-RAS4A with higher affinity than to K-RAS4B; the same was observed for Raf1 (Zhang et al., 2018; Amendola et al., 2019). Part of the figure was built and adapted from the "Ras Pathway" template, by BioRender.com.

colleagues proposed that the N-RAS-like state of K-RAS4A (i.e., palmitoylated and farnesylated) could influence its high expression in melanoma, and that the K-RAS4B-like state of K-RAS4A (i.e., farnesylated) could contribute to the high expression levels seen in colon cancer (Nussinov et al., 2016).

Regarding benign tumour tissues, Shahrabi-Farahani and colleagues reported that during the proliferative phase of the menstrual cycle, K-RAS4A mRNA was upregulated (2.7-fold higher) in eutopic endometrium in patients with endometriosis compared to controls (Shahrabi-Farahani et al., 2015), whereas no significant correlation was observed between K-RAS4B and the different menstrual cycle phases (Farahani et al., 2015). Shahrabi-Farahani and colleagues proposed that increasing the K-RAS4A $\backslash 4 \mathrm{~B}$ ratio could affect the equilibrium between proliferation and apoptosis, two processes that are responsible for maintaining a normal eutopic endometrium, thus leading to the proliferative phase defect seen in patients with endometriosis. Furthermore, expression of both splice variants was also detected in patients with leiomyoma (i.e., uterine tumours originating from smooth muscle cells) (Zolfaghari et al., 2017).

\section{POSSIBLE ROLES FOR K-RAS4A IN TUMORIGENESIS}

Different roles have been attributed to K-RAS4A. Studies of embryonic stem cells have suggested that K-RAS4A promotes 
apoptosis while K-RAS4B inhibits it, and that the K-RAS4A/4B isoform ratio regulates tumorigenesis by influencing stem cell differentiation and survival (Plowman et al., 2006). In addition, K-RAS4A was recently shown to be enriched in cancer stem-like cells under hypoxia conditions, whereas K-RAS4B was mainly induced by ER stress (Chen et al., 2021). Chen and colleagues also suggested that K-RAS4A splicing could be controlled by the DCAF15/RBM39 pathway (Chen et al., 2021). Another study used a matrix metalloproteinase 2 (MMP-2) promoter-luciferase reporter assay to demonstrate that the transcription of MMP-2 in K-RAS knockout fibroblasts was partially restored by transient expression of K-RAS4B but not K-RAS4A (Liao et al., 2003). This finding suggests that K-RAS4B has a greater metastatic potential than K-RAS4A, because tumour cells that express oncogenic RAS have a higher metastatic potential partially due to up-regulation of MMP-2 (Liao et al., 2003). Overall, both reports support a more tumorigenic role for K-RAS4B than K-RAS4A.

Interestingly, K-RAS4A shares similarities with H-RAS; both have been shown to induce lung tumours in wildtype and H-RAS knock-in mice (To et al., 2008). Since K-RAS presents mutations at the same regions in both splice variants, the vast majority affecting codon G12, some cancers may harbour mutations in one or even both isoforms simultaneously. Thus, blocking one isoform might not be enough to fully reduce the cell's oncogenic potential. Oncogenic K-RAS4A has also been shown to induce lung carcinogenesis in mice (To et al., 2008), and a recent publication by the Barbacid group demonstrated that expression of $\mathrm{K}-\mathrm{RAS} 4 \mathrm{~A}^{\mathrm{G} 12 \mathrm{~V}}$ in mice that lack K-RAS4B is sufficient to promote metastatic lung adenocarcinomas (Salmon et al., 2021). These reports highlight K-RAS4A's oncogenic potential, suggesting it could serve as a future therapeutic target.

Studies performed on patient samples have also supported different roles for each isoform. Abubaker and colleagues found an association between K-RAS4A overexpression and improved overall survival in patients with colorectal cancer, whereas overexpression of K-RAS4B was significantly associated with larger tumour size (Abubaker et al., 2009). The RAS oncogene is also involved in cell metabolism, and it was suggested that distinct RAS mutations might lead to variable metabolic dependencies (Kimmelman 2015). Recently, hexokinase 1 (HK1) was shown to be a K-RAS4A effector, which could impact on the tumours' cells metabolism (Amendola et al., 2019).

In human K-RAS-mutant leukaemia cell lines and in acute myeloid leukaemia (AML) cells, K-RAS4A is also expressed, and Zhao and colleagues showed that cells harbouring mutations at the palmitoylation site of oncogenic K-RAS4A (i.e., palmitoylation-defective mutant K-RAS4A ${ }^{\mathrm{G} 12 \mathrm{D} / \mathrm{C} 180 \mathrm{~S}}$ ) present a reduction in leukemogenicity potential. Unlike the results seen with mutations at the palmitoylation site of N-RAS (i.e., palmitoylation-defective mutant N-RAS ${ }^{\text {G12D/C181S }}$, palmitoylation-defective K-RAS4A could still induce leukaemia in mice (Zhao et al., 2015). The KIKK motif of K-RAS4A appears to impact on its transforming activity since mutations affecting both the palmitoylation site and the KIKK motif blocked oncogenic K-RAS4A from inducing leukaemia in mice (Zhao et al., 2015). These findings support a role for the different posttranslational modifications in RAS function and oncogenic potential.

The fact that both splice variants are identical in the region where most K-RAS oncogenic mutations occur suggests that previous reports of mutations in K-RAS may actually have uncovered mutations in both transcripts, not just in K-RAS4B. In addition, cancers harbouring K-RAS mutations may behave differently depending on which splice variant is predominantly affected, which could impact on therapy response. As K-RAS4A and K-RAS4B possess slightly different structures when in the GDPbound state, with GDP-bound K-RAS4A presenting a more exposed nucleotide binding pocket than GDP-bound K-RAS4B (Chakrabarti et al., 2016), compounds developed to target this catalytic domain could also be considered as a means to differentiate between the oncogenic mutant variants. The recent FDA approval of Sotorasib or Lumakras (previously known as AMG 510, Amgen), a K-RAS ${ }^{\mathrm{G} 12 \mathrm{C}}$ inhibitor able to reduce K-RAS ${ }^{\mathrm{G} 12 \mathrm{C}}$ tumours (Canon et al., 2019; Hong et al., 2020), is a major breakthrough in RAS biology, since for many years RAS was considered an undruggable target. How efficient this drug is when comparing K-RAS4A ${ }^{\mathrm{G} 12 \mathrm{C}}$ versus K-RAS4B ${ }^{\mathrm{G} 12 \mathrm{C}}$ in different cancer types remains to be determined. It would be interesting to see the development of novel mutation- and splice variant-specific inhibitors in those cancers where both isoforms are simultaneously affected. Nevertheless, more analysis should be performed to better clarify if there are any significant differences between mutant K-RAS4A and mutant K-RAS4B in response to distinct therapies.

\section{CONCLUSION}

K-RAS4B research has historically overshadowed that of K-RAS4A, suggesting that K-RAS4A is a minor variant. Nevertheless, the fact that K-RAS4A is evolutionarily conserved and binds distinct effectors at different affinities compared to K-RAS4B, in addition to the fact that K-RAS4A expression varies across tissue types, argue for a more important role than previously thought. Additional work is needed to unravel the different roles that each splice variant plays in normal versus tumours tissues. Such knowledge may help inform understanding of therapy resistance and improve disease management of cancer types with differential splice variant expression. Personalised medicine has exploited K-RAS-mutationspecific tumour differences for the development of mutationselective anti-RAS strategies; thus, it could be beneficial to place K-RAS4A in the spotlight and perhaps achieve more selective cancer treatment strategies.

\section{AUTHOR CONTRIBUTIONS}

VA contributed to conception, writting and design of the study.

\section{ACKNOWLEDGMENTS}

I would like to thank Dr Lindsay Carpp and Dr Marion S Gilbert for proofreading the final version of this manuscript. 


\section{REFERENCES}

Abubaker, J., Bavi, P., Al-Haqawi, W., Sultana, M., Al-Harbi, S., Al-Sanea, N., et al. (2009). Prognostic Significance of Alterations in KRAS Isoforms KRAS-4A/4B andKRASmutations in Colorectal Carcinoma. J. Pathol. 219, 435-445. doi:10.1002/path.2625

Ahearn, I., Zhou, M., and Philips, M. R. (2018). Posttranslational Modifications of RAS Proteins. Cold Spring Harb Perspect. Med. 8. doi:10.1101/ cshperspect.a031484

Amendola, C. R., Mahaffey, J. P., Parker, S. J., Ahearn, I. M., Chen, W.-C., Zhou, M., et al. (2019). KRAS4A Directly Regulates Hexokinase 1. Nature 576, 482-486. doi:10.1038/s41586-019-1832-9

Aran, V., Masson Domingues, P., Carvalho de Macedo, F., Moreira de Sousa, C. A., Caldas Montella, T., de Souza Accioly, M. T., et al. (2018). A Cross-Sectional Study Examining the Expression of Splice Variants K-Ras4a and K-Ras $4 \mathrm{~b}$ in Advanced Non-small-cell Lung Cancer Patients. Lung Cancer 116, 7-14. doi:10.1016/j.lungcan.2017.12.005

Barceló, C., Paco, N., Morell, M., Alvarez-Moya, B., Bota-Rabassedas, N., Jaumot, M., et al. (2014). Phosphorylation at Ser-181 of Oncogenic KRAS Is Required for Tumor Growth. Cancer Res. 74, 1190-1199. doi:10.1158/0008-5472.can-131750

Bigenzahn, J. W., Collu, G. M., Kartnig, F., Pieraks, M., Vladimer, G. I., Heinz, L. X., et al. (2018). LZTR1 Is a Regulator of RAS Ubiquitination and Signaling. Science 362, 1171-1177. doi:10.1126/science.aap8210

Canon, J., Rex, K., Saiki, A. Y., Mohr, C., Cooke, K., Bagal, D., et al. (2019). The Clinical KRAS(G12C) Inhibitor AMG 510 Drives Anti-tumour Immunity. Nature 575, 217-223. doi:10.1038/s41586-019-1694-1

Capon, D. J., Seeburg, P. H., McGrath, J. P., Hayflick, J. S., Edman, U., Levinson, A. D., et al. (1983). Activation of Ki-Ras2 Gene in Human colon and Lung Carcinomas by Two Different point Mutations. Nature 304, 507-513. doi:10.1038/304507a0

Chakrabarti, M., Jang, H., and Nussinov, R. (2016). Comparison of the Conformations of KRAS Isoforms, K-Ras4A and K-Ras4B, Points to Similarities and Significant Differences. J. Phys. Chem. B 120, 667-679. doi:10.1021/acs.jpcb.5b11110

Chen, W.-C., To, M. D., Westcott, P. M. K., Delrosario, R., Kim, I.-J., Philips, M., et al. (2021). Targeting KRAS4A Splicing through the RBM39/DCAF15 Pathway Inhibits Cancer Stem Cells. Nat. Commun. 12, 4288. doi:10.1038/ s41467-021-24498-7

Cho, K.-j., van der Hoeven, D., Zhou, Y., Maekawa, M., Ma, X., Chen, W., et al. (2016). Inhibition of Acid Sphingomyelinase Depletes Cellular Phosphatidylserine and Mislocalizes K-Ras from the Plasma Membrane. Mol. Cel Biol 36, 363-374. doi:10.1128/mcb.00719-15

Cox, A. D., and Der, C. J. (2010). Ras History. Small GTPases 1, 2-27. doi:10.4161/ sgtp.1.1.12178

Esteban, L. M., Vicario-Abejo'n, C., Ferna'ndez-Salguero, P., Ferna'ndezMedarde, A., Swaminathan, N., Yienger, K., et al. (2001). Targeted Genomic Disruption of H- Ras and N- Ras, Individually or in Combination, Reveals the Dispensability of Both Loci for Mouse Growth and Development. Mol. Cel Biol 21, 1444-1452. doi:10.1128/mcb.21.5.1444-1452.2001

Farahani, M. S., Shahbazi, S., Moghaddam, S. A., and Mahdian, R. (2015). Evaluation of KRAS Gene Expression and LCS6 Variant in Genomic and Cell-free DNA of Iranian Women with Endometriosis. Reprod. Sci. 22, 679-684. doi:10.1177/1933719114556478

Hancock, J. F., Cadwallader, K., Paterson, H., and Marshall, C. J. (1991). A CAAX or a CAAL Motif and a Second Signal Are Sufficient for Plasma Membrane Targeting of Ras Proteins. EMBO J. 10, 4033-4039. doi:10.1002/j.14602075.1991.tb04979.x

Hancock, J. F., Paterson, H., and Marshall, C. J. (1990). A Polybasic Domain or Palmitoylation Is Required in Addition to the CAAX Motif to Localize P21 ras to the Plasma Membrane. Cell 63, 133-139. doi:10.1016/0092-8674(90) 90294-o

Hancock, J. F. (2003). Ras Proteins: Different Signals from Different Locations. Nat. Rev. Mol. Cel Biol 4, 373-385. doi:10.1038/nrm1105

Hobbs, G. A., Der, C. J., and Rossman, K. L. (2016). RAS Isoforms and Mutations in Cancer at a Glance. J. Cel Sci 129, 1287-1292. doi:10.1242/jcs.182873
Hong, D. S., Fakih, M. G., Strickler, J. H., Desai, J., Durm, G. A., Shapiro, G. I., et al. (2020). KRASG12C Inhibition with Sotorasib in Advanced Solid Tumors. N. Engl. J. Med. 383, 1207-1217. doi:10.1056/nejmoa1917239

James, G., Goldstein, J. L., and Brown, M. S. (1996). Resistance of K-RasBV12 Proteins to Farnesyltransferase Inhibitors in Rat1 Cells. Proc. Natl. Acad. Sci. 93, 4454-4458. doi:10.1073/pnas.93.9.4454

Jing, H., Zhang, X., Wisner, S. A., Chen, X., Spiegelman, N. A., Linder, M. E., et al. (2017). 'SIRT2 and Lysine Fatty Acylation Regulate the Transforming Activity of K-Ras4a, Elife 6. doi:10.7554/elife.32436

Kelemen, O., Convertini, P., Zhang, Z., Wen, Y., Shen, M., Falaleeva, M., et al. (2013). Function of Alternative Splicing. Gene 514, 1-30. doi:10.1016/ j.gene.2012.07.083

Kimmelman, A. C. (2015). Metabolic Dependencies in RAS-Driven Cancers. Clin. Cancer Res. 21, 1828-1834. doi:10.1158/1078-0432.ccr-14-2425

King, S., Bray, S., Galbraith, S., Christie, L., and Fleming, S. (2014). Evidence for Aldosterone-dependent Growth of Renal Cell Carcinoma. Int. J. Exp. Path. 95, 244-250. doi:10.1111/iep.12074

Koera, K., Nakamura, K., Nakao, K., Miyoshi, J., Toyoshima, K., Hatta, T., et al. (1997). K-ras Is Essential for the Development of the Mouse Embryo. Oncogene 15, 1151-1159. doi:10.1038/sj.onc.1201284

Konstantinopoulos, P. A., Karamouzis, M. V., and Papavassiliou, A. G. (2007). Post-translational Modifications and Regulation of the RAS Superfamily of GTPases as Anticancer Targets. Nat. Rev. Drug Discov. 6, 541-555. doi:10.1038/ $\operatorname{nrd} 2221$

Laude, A. J., and Prior, I. A. (2008). Palmitoylation and Localisation of RAS Isoforms Are Modulated by the Hypervariable Linker Domain. J. Cel Sci 121, 421-427. doi:10.1242/jcs.020107

Li, Z.-L., and Buck, M. (2017). Computational Modeling Reveals that Signaling Lipids Modulate the Orientation of K-Ras4A at the Membrane Reflecting Protein Topology. Structure 25, 679-689. doi:10.1016/j.str.2017.02.007

Liao, J., Wolfman, J. C., and Wolfman, A. (2003). K-ras Regulates the Steady-State Expression of Matrix Metalloproteinase 2 in Fibroblasts. J. Biol. Chem. 278, 31871-31878. doi:10.1074/jbc.m301931200

Marks, J. L., Broderick, S., Zhou, Q., Chitale, D., Li, A. R., Zakowski, M. F., et al. (2008). Prognostic and Therapeutic Implications of EGFR and KRAS Mutations in Resected Lung Adenocarcinoma. J. Thorac. Oncol. 3, 111-116. doi:10.1097/jto.0b013e318160c607

McGrath, J. P., Capon, D. J., Smith, D. H., Chen, E. Y., Seeburg, P. H., Goeddel, D. V., et al. (1983). Structure and Organization of the Human Ki-Ras ProtoOncogene and a Related Processed Pseudogene. Nature 304, 501-506. doi:10.1038/304501a0

Messina, S., De Simone, G., and Ascenzi, P. (2019). Cysteine-based Regulation of Redox-Sensitive Ras Small GTPases. Redox Biol. 26, 101282. doi:10.1016/ j.redox.2019.101282

Murugan, A. K., Grieco, M., and Tsuchida, N. (2019). RAS Mutations in Human Cancers: Roles in Precision Medicine. Semin. Cancer Biol. 59, 23-35. doi:10.1016/j.semcancer.2019.06.007

Newlaczyl, A. U., Coulson, J. M., and Prior, I. A. (2017). Quantification of Spatiotemporal Patterns of Ras Isoform Expression during Development. Sci. Rep. 7, 41297. doi:10.1038/srep41297

Normanno, N., Tejpar, S., Morgillo, F., De Luca, A., Van Cutsem, E., and Ciardiello, F. (2009). Implications for KRAS Status and EGFR-Targeted Therapies in Metastatic CRC. Nat. Rev. Clin. Oncol. 6, 519-527. doi:10.1038/ nrclinonc.2009.111

Nussinov, R., Tsai, C.-J., Chakrabarti, M., and Jang, H. (2016). A New View of Ras Isoforms in Cancers. Cancer Res. 76, 18-23. doi:10.1158/0008-5472.can-151536

Pao, W., Wang, T. Y., Riely, G. J., Miller, V. A., Pan, Q., Ladanyi, M., et al. (2005). KRAS Mutations and Primary Resistance of Lung Adenocarcinomas to Gefitinib or Erlotinib. Plos Med. 2, e17. doi:10.1371/journal.pmed.0020017

Pells, S., Divjak, M., Romanowski, P., Impey, H., Hawkins, N. J., Clarke, A. R., et al. (1997). Developmentally-regulated Expression of Murine K-Ras Isoforms. Oncogene 15, 1781-1786. doi:10.1038/sj.onc.1201354

Plowman, S., Arends, M., Brownstein, D., Luo, F., Devenney, P., Rose, L., et al. (2006). The K-Ras 4A Isoform Promotes Apoptosis but Does Not Affect Either Lifespan or Spontaneous Tumor Incidence in Aging Mice. Exp. Cel Res. 312, 16-26. doi:10.1016/j.yexcr.2005.10.004 
Plowman, S. J., Williamson, D. J., O’Sullivan, M. J., Doig, J., Ritchie, A.-M., Harrison, D. J., et al. (2003). While K- Ras Is Essential for MouseDevelopment, Expression of the K- Ras 4A Splice VariantIsDispensable. Mol. Cel Biol 23, 9245-9250. doi:10.1128/ mcb.23.24.9245-9250.2003

Salmón, M., Paniagua, G., Lechuga, C. G., Fernández-García, F., Zarzuela, E., Álvarez-Díaz, R., et al. (2021). KRAS4A Induces Metastatic Lung Adenocarcinomas In Vivo in the Absence of the KRAS4B Isoform. Proc. Natl. Acad. Sci. U S A., 118. doi:10.1073/pnas.2023112118

Shahrabi-Farahani, M., Shahbazi, S., Mahdian, R., and Amini-Moghaddam, S. (2015). K-ras 4A Transcript Variant Is Up-Regulated in Eutopic Endometrium of Endometriosis Patients during Proliferative Phase of Menstrual Cycle. Arch. Gynecol. Obstet. 292, 225-229. doi:10.1007/s00404-014-3596-7

Shimizu, K., Birnbaum, D., Ruley, M. A., Fasano, O., Suard, Y., Edlund, L., et al. (1983). Structure of the Ki-Ras Gene of the Human Lung Carcinoma Cell Line Calu-1. Nature 304, 497-500. doi:10.1038/304497a0

Sorek, R., and Amitai, M. (2001). Piecing Together the Significance of Splicing. Nat. Biotechnol. 19, 196. doi:10.1038/85613

Spiegelman, N. A., Hong, J. Y., Hu, J., Jing, H., Wang, M., Price, I. R., et al. (2019). A Small-Molecule SIRT2 Inhibitor that Promotes K-Ras4a Lysine FattyAcylation. ChemMedChem 14, 744-748. doi:10.1002/cmdc.201800715

Stalnecker, C. A., and Der, C. J. (2020). RAS, Wanted Dead or Alive: Advances in Targeting RAS Mutant Cancers. Sci. Signal. 13. doi:10.1126/scisignal.aay6013

To, M. D., Wong, C. E., Karnezis, A. N., Del Rosario, R., Di Lauro, R., and Balmain, A. (2008). Kras Regulatory Elements and Exon 4A Determine Mutation Specificity in Lung Cancer. Nat. Genet. 40, 1240-1244. doi:10.1038/ng.211

Tsai, F. D., Lopes, M. S., Zhou, M., Court, H., Ponce, O., Fiordalisi, J. J., et al. (2015). K-Ras4A Splice Variant Is Widely Expressed in Cancer and Uses a Hybrid Membrane-Targeting Motif. Proc. Natl. Acad. Sci. USA 112, 779-784. doi:10.1073/pnas.1412811112

Voice, J. K., Klemke, R. L., Le, A., and Jackson, J. H. (1999). Four Human Ras Homologs Differ in Their Abilities to Activate Raf-1, Induce Transformation, and Stimulate Cell Motility. J. Biol. Chem. 274, 17164-17170. doi:10.1074/ jbc.274.24.17164
Wright, L. P., and Philips, M. R. (2006). Thematic Review Series: Lipid Posttranslational Modifications CAAX Modification and Membrane Targeting of Ras. J. Lipid Res. 47, 883-891. doi:10.1194/jlr.r600004-jlr200

Zhang, X., Cao, J., Miller, S. P., Jing, H., and Lin, H. (2018). Comparative Nucleotide-dependent Interactome Analysis Reveals Shared and Differential Properties of KRas4a and KRas4b. ACS Cent. Sci. 4, 71-80. doi:10.1021/ acscentsci.7b00440

Zhao, H., Liu, P., Zhang, R., Wu, M., Li, D., Zhao, X., et al. (2015). Roles of Palmitoylation and the KIKK Membrane-Targeting Motif in Leukemogenesis by Oncogenic KRAS4A. J. Hematol. Oncol. 8, 132. doi:10.1186/s13045-015-0226-1

Zhou, Y., Prakash, P., Gorfe, A. A., and Hancock, J. F. (2018). Ras and the Plasma Membrane: A Complicated Relationship. Cold Spring Harb Perspect. Med. 8. doi:10.1101/cshperspect.a031831

Zolfaghari, N., Shahbazi, S., Torfeh, M., Khorasani, M., Hashemi, M., and Mahdian, R. (2017). Identification of Differentially Expressed K-Ras Transcript Variants in Patients with Leiomyoma. Reprod. Sci. 24, 1438-1443. doi:10.1177/1933719116689596

Conflict of Interest: The author declares that the research was conducted in the absence of any commercial or financial relationships that could be construed as a potential conflict of interest.

Publisher's Note: All claims expressed in this article are solely those of the authors and do not necessarily represent those of their affiliated organizations, or those of the publisher, the editors and the reviewers. Any product that may be evaluated in this article, or claim that may be made by its manufacturer, is not guaranteed or endorsed by the publisher.

Copyright (c) 2021 Aran. This is an open-access article distributed under the terms of the Creative Commons Attribution License (CC BY). The use, distribution or reproduction in other forums is permitted, provided the original author(s) and the copyright owner(s) are credited and that the original publication in this journal is cited, in accordance with accepted academic practice. No use, distribution or reproduction is permitted which does not comply with these terms. 